\title{
Dynamic Changes of Sp6 Transgene Expression in Dental Epithelial Cells during Long-term Culture
}

\author{
Trianna W. Utami \\ Keiko Miyoshi \\ Hiroko Hagita \\ Ryna D. Yanuaryska \\ Taigo Horiguchi \\ Takafumi Noma
}

\begin{abstract}
Department of Molecular Biology, Institute of Health Biosciences, The University of Tokushima Graduate School, Tokushima, Japan

E-mail:ntaka@tokushima-u.ac.jp

Received April 11, 2011; Accepted May 11, 2011
\end{abstract}

\begin{abstract}
To investigate the function of specificity protein 6 (SP6) transcription factor by gain-of-function procedure, we established cytomegalovirus (CMV) promoter-driven Sp6 stable transformants, C9 cells, using dental epithelialderived cells. Initially, C9 cells produced a significant amount of SP6 protein. However, SP6 expression was reduced in these cells upon long-term culture. We could detect $S p 6$ transcripts in C 9 cells by RT-PCR throughout the passages, although the CMV promoter is known to be epigenetically silenced. We recently found that SP6 was a short-lived protein that was degraded by a ubiquitin-independent proteasome pathway, although it is yet unclear how Sp6 expression was regulated during culture. Thus, we studied the possibility of epigenetic regulation of Sp6 expression. Comparative analysis of endogenous and exogenous Sp6 mRNA expressions demonstrated the specific down-regulation of exogenous Sp6 mRNA levels during culture passages. A DNA methyltransferase inhibitor, 5-Aza-2'-deoxycytidine (5AC), and a histone deacetylase inhibitor, valproic acid (VPA), enhanced or induced SP6 protein expression up to passage 28 without enhancing the mRNA level. The dramatic up-regulation of exogenous Sp6 mRNA was uniquely observed only at passage 50 by 5 AC or VPA treatment. These findings indicate that multiple epigenetic regulatory mechanisms operate to fine-tune $\operatorname{Sp} 6$ expression during long-term culture.
\end{abstract}

Keywords: DNA methylation, epigenetic regulation, gain-of-function, histone modification, Sp6

\section{Introduction}

Tooth development is a multistep process that is regulated by a combination of genetic and epigenetic information ${ }^{1,2}$. Numerous transcription factors have been identified that are involved in this developmental process ${ }^{3,4}$. Sp6, also known as epiprofin, was first identified in dental epithelial cells and belongs to the SP/KLF transcription factor family ${ }^{5-7}$. It is one of the critical molecules required for tooth development ${ }^{4,8}$, as $S p 6$ deficientmice show abnormal tooth phenotypes, such as delayed eruption of incisors, supernumerary teeth, or enamel disorganizations, in addition to other 
abnormal tissue phenotypes, including hair loss, lung abnormalities, and limb deformities ${ }^{9,10}$. However, the molecular mechanisms underlying these Sp6deficient phenotypes remain unclear.

In a previous study, we found that overexpression of the Sp6 gene inhibited follistatin (Fst) gene expression in dental epithelial cells, suggesting that SP6 regulated tooth development by modulating bone morphogenetic protein (BMP) signaling ${ }^{11}$. We subsequently characterized the structure of the Sp6 gene and detected two alternative promoter activities in a cell-type specific manner in addition to a possible third promoter activity located between exon $1 \mathrm{~b}$ and exon 2 in dental epithelial cells ${ }^{12}$. At the same time, we found that Sp6 expression was up-regulated by both BMP and Wnt signals, which provided a possible linkage between cytokine regulation of Sp 6 expression and inductive epithelialmesenchymal interactions through a feedback loop involving BMP signaling during tooth development 11-13. We also found that the SP6 protein is shortlived and degraded by a ubiquitin-independent proteasome pathway ${ }^{14}$. These findings suggested that fine-tuning of $S p 6$ expression is required during tooth development.

In this study, we analyzed the epigenetic regulation of $S p 6$ gene expression in C9 cells treated with a DNA methyltransferase inhibitor (DNMTI), $5 A C^{15}$, and a histone deacetylase inhibitor (HDACl), $V_{P A}{ }^{16}$. C9 is a Sp6 stable transformant clone that is driven by the cytomegalovirus (CMV) promoter. High SP6 protein expression in C9 cells was detected at passage 7 (P7) but not at P28 and P50. When we treated $C 9$ cells with the epigenetic regulatory reagents, protein expression was enhanced or induced at P7 and P28 but not at P50. In contrast, we found no enhancement of either exogenous or endogenous Sp6 mRNA at any cell passages, except for exogenous Sp 6 mRNA at P50 after treatment with $5 A C$ and VPA, while Sp6 transcripts were detected throughout the cell passages. These findings suggest that the epigenetic status tightly regulates not only Sp6 gene expression but also SP6 protein expression in C9 cells during long-term culture. Our findings provide a new perspective for the regulation of the Sp6 mRNA and protein levels to understand the SP6 function in tooth development.

\section{Materials and Methods}

\subsection{Cell line and culture conditions}

The SP6 high producer CHA9 cells was originally established as described previously ${ }^{11}$. Briefly, an HAtagged SP6 coding sequence (CHA-Sp6) was inserted into a CMV promoter-driven expression plasmid. Then, the expression plasmid was transfected into dental epithelial, ameloblast lineage clone, G5 cells $^{17}$. CHA9 cells were selected by serial limiting dilutions in the presence of $\mathrm{G} 418(400 \mu \mathrm{g} / \mathrm{ml}$ : Nakalai Tesque, Kyoto, Japan) followed by Western blot analysis to identify the highest SP6 producer. C9 cells were then recloned from $\mathrm{CHA} 9$ cells because SP6 expression was silenced. In the same manner as $\mathrm{CHA} 9$ cells, we performed serial limiting dilutions in the presence of $\mathrm{G} 418(400 \mu \mathrm{g} / \mathrm{ml})$ and Western blot analysis to isolate a Sp6 high producer clone, which was designated as C9. For maintenance, these cells were cultured in a combination of Dulbecco's modified Eagle's medium/Ham's F12 (D/ F12) medium, supplemented with $10 \%$ fetal bovine serum (JRH Biosciences, Lenexa, KS) and $400 \mu \mathrm{g} / \mathrm{ml}$ of G418.

\subsection{Reagents}

5AC and VPA were purchased from Sigma-Aldrich, Inc. (St. Louis, MO, USA) and Wako Pure Chemical Industries, Ltd. (Osaka, Japan), respectively. These reagents were added to cultures of C9 cells at $70 \%$ confluence and cultured for the indicated time.

\subsection{Western Blot analysis}

C9 cells were harvested and lysed in $2 x$ sodium dodecyl sulfate (SDS) sample buffer as described previously ${ }^{11}$. Protein concentration was determined using the BCA Protein Assay Kit (Pierce, Rockford, IL, USA). Total protein ( $30 \mu \mathrm{g})$ was loaded onto a $10 \%$ SDS-polyacrylamide gel. After electrophoresis, proteins were transferred to a polyvinylidene difluoride (PVDF) membrane (ImmobilonTM; Millipore, Bedford, MA, USA) and blocked with 5\% skim milk in tris-buffered saline (TBS: $10 \mathrm{mM}$ Tris$\mathrm{HCl}, \mathrm{pH}$ 8.0, $150 \mathrm{mM} \mathrm{NaCl}$ ) with 0.05\% Tween-20 
for 3 hours at room temperature. The membranes were incubated with an anti-HA antibody (1:5000; Santa Cruz Biotechnology, Santa Cruz, CA, USA) overnight, following which immunodetection was performed. Quantification of detected signals was performed by densitometric analysis using "Quantity One" software (Bio-Rad laboratories, Hercules, CA, USA) according to the manufacturer's instructions. Lysates of Cos7 cells transfected with CHA-Sp6 in $\mathrm{pClneo}$ were used as a positive control.

\subsection{Reverse transcription-PCR (RT-PCR) and quantitative RT-PCR analysis}

Total RNA was isolated using TriReagent (Molecular Research Center. Inc., Cincinnati, OH, USA) according to the manufacturer's instructions. After treatment with DNase I (Invitrogen, Carlsbad, CA, USA), 500 ng of total RNA was converted into CDNA using an RNA PCR kit AMV Ver.3.0 (Takara, Shiga, Japan) according to the manufacturer's instructions. Synthesized cDNA was used for either PCR analysis using Taq DNA polymerase (Promega, Madison WI, USA) or quantitative RT-PCR (qRT-PCR) using THUNDERBIRD ${ }^{\text {TM }}$ SYBR qPCR Mix (Toyobo Co. Ltd., Osaka, Japan). qRT-PCR was performed using a 7300 Real-Time PCR system (Applied Biosystems, Carlsbad, CA, USA). Expression level ratios were calculated from the threshold cycles and normalized to those of $18 \mathrm{~s}$ rRNA levels. All results were confirmed by triplicate experiments. Gene specific primer sets are shown in Table 1.

\subsection{Statistics}

RT-PCR and qRT-PCR results are from three independent analyses performed under the same experimental conditions. Results are presented as means \pm S.D. of triplicate samples for the representative experiments.

\section{Results}

\subsection{SP6 protein expression during long-term culture}

To investigate the SP6 function, we originally established Sp6 stable transformant $\mathrm{CHA9}$ cells ${ }^{11}$ using dental epithelial-derived $\mathrm{G} 5$ cells ${ }^{16}$. Unexpectedly, we found that the SP6 protein expression level was reduced in CHA9 cells during long-term culture. Therefore, we recloned a new SP6 high producer clone from $\mathrm{CHA} 9$ cells, designated as C9 cells. At P28, we again found a significant reduction of SP6 protein expression in C9 cells compared with the high SP6 expression at P7 (Figure 1A).
A

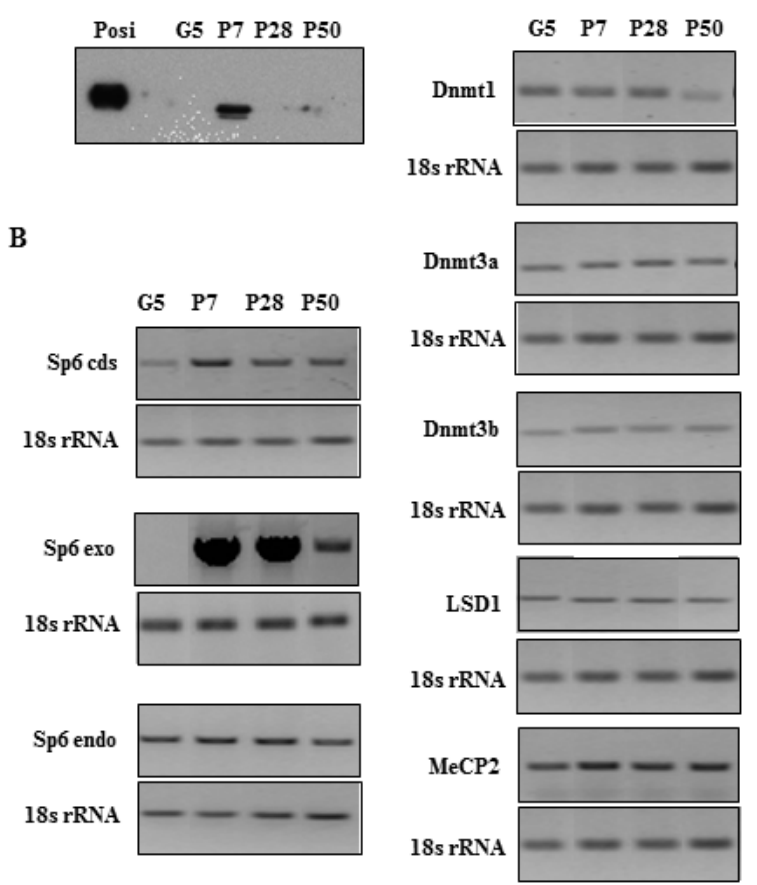

Figure 1. SP6 protein expression during long-term culture. (A) SP6 expression in C9 (P7, P28, and P50) and parental G5 cells. The levels of SP6 protein expression were analyzed by Western Blots using an anti-HA antibody. Posi: positive control. (B) Sp6 mRNA expression in C9 (P7, P28, and P50) and G5 cells. Sp6 cds: coding region of Sp6; Sp6 endo: endogenous Sp6; Sp6 exo: exogenous Sp6. (C) Epigenetic-related gene expressions in C9 (P7, P28, and P50) and G5 cells. 
Table 1. Gene Specific Primers

\begin{tabular}{|c|c|}
\hline Gene & Primer sequence \\
\hline \multirow{2}{*}{ 18s rRNA } & 5'CTACCTGGTTGATCCTGCCAGTAGGATC3' \\
\hline & $5^{\prime}$ CCCCGTCGGCATGTATTAGCTCTAGAAC3' \\
\hline \multirow{2}{*}{ Dnmt1 } & $5^{\prime}$ CGCTAAGGACGATGATGAGACGCC ${ }^{\prime}$ \\
\hline & 5'CCTTTTTGGGTGACGGCAACTCC3' \\
\hline \multirow{2}{*}{ Dnmt3a } & 5'CCAGCGTCACACAGAAGCATATCCC3' \\
\hline & 5'CGGTCCTCACTTTGCTGAACTTGGC3' \\
\hline \multirow{2}{*}{ Dnmt3b } & 5'CGCAGGAAACCATGAAGGGAGC(3' \\
\hline & 5'CCCCTCTTAGACAGCCCTGAGC(3' \\
\hline \multirow{2}{*}{ LSD1 } & 5'CACACACGCTCCACAAGTCAGC3' \\
\hline & 5'CGCCGACAATCACATCATCAC \\
\hline \multirow{2}{*}{ MeCP2 } & 5'CAGAGGGCAAACATGAACCACC3' \\
\hline & 5'CGAGGTGTCTCCCACCTTTTCC ${ }^{\prime}$ \\
\hline \multirow{2}{*}{ Sp6 cds } & 5'CCCGGCAATGCTAACCGCTGTCTGTGC3' \\
\hline & 5'CGGCTCAGTTGGAGGACGCCGAGCTGC(3' \\
\hline \multirow{2}{*}{$\begin{array}{c}\text { Sp6 } \\
\text { endogenous }\end{array}$} & 5'CGCGCCATCTTCAGACCCACC ${ }^{\prime}$ \\
\hline & 5'CCCACTTCGCAAGAGGATTTCC ${ }^{\prime}$ \\
\hline \multirow{2}{*}{$\begin{array}{c}\text { Sp6 } \\
\text { exogenous }\end{array}$} & 5'CGGCTAGAGTACTTAATACGACTCACC3' \\
\hline & 5'CCTCGAAGCATTAACCCTCACTAAAGC(3' \\
\hline
\end{tabular}

To determine the cause of this reduced expression, we first examined the levels of $S p 6$ transcripts in $C 9$ cells using specific primers to distinguish between the three types of Sp6 mRNA: total (coding sequence; cds), exogenous, and endogenous Sp6 mRNAs. We found that the exogenous Sp6 mRNA level was remarkably high at P7 and P28 but reduced at P50. However, the endogenous Sp6 expression levels were not changed throughout the cell passages (Figure 1B).

Based on these findings, we hypothesized that epigenetic mechanisms may have been involved in Sp6 transgene expression in C9 cells at P50, and we further analyzed the mRNA expression levels of epigenetic-related genes in C9 cells: DNA methyltransferase 1(Dnmt1), DNA methyltransferase 3a (Dnmt3a), DNA methyltransferase 3b (Dnmt3b), methyl-CpG-binding protein-2 (MeCP2), and lysinespecific demethylase 1 (LSD1) ${ }^{[18-20]}$. As shown in Figure 1C, similar expression levels were detected for these genes at each passage, except for Dnmt1. The level of Dnmt1 transcripts was decreased at P50 three to fourfold compared to the levels at P7 or P28.

\subsection{Responsiveness of C9 cells to 5AC and VPA}

To further elucidate the epigenetic regulation of $S p 6$ mRNA and SP6 protein expression during long-term culture, we treated $C 9$ cells with $5 A C$ and VPA. Both $5 A C$ and VPA enhanced or induced the SP6 protein expression at P7 and P28 in a dose- and timedependent manner (Figures 2A and 2B). However, SP6 protein was not detected in C9 cells at P50 with either $5 \mathrm{AC}$ or VPA treatment (Figure 2B).

Next, we examined whether Sp6 mRNA levels were enhanced in response to $5 A C$ and VPA (Figure 2C). Unexpectedly, we found no dramatic changes in the levels of endogenous Sp6 mRNA with either reagent. In contrast, the mRNA level of exogenous Sp6 at P50 showed over 3-fold enhancement with either 5AC or VPA treatment compared to the controls. At P7 and P28, the levels of exogenous Sp6 mRNA expression showed a tendency to be slightly or marginally increased compared to the controls in response to both treatments.

\section{3 qPCR analysis of epigenetic-related gene expression in response to $5 A C$ and VPA}

To investigate whether the selective enhancement of Sp6 mRNA expression was correlated with the expression of epigenetic-related genes, we analyzed their expressions in response to 5AC and VPA. As shown in Figure 3, Dnmt1, Dnmt3a, Dnmt3b, LSD1, and MeCP2 expressions were all decreased to less than $20 \%$ of those in controls at P7 and P28 in response to $5 A C$ and VPA treatment. However, the responsiveness of these genes to $5 \mathrm{AC}$ and VPA either disappeared or was reduced at P50, except for $L S D 1$. In addition, the basal expression levels of these epigenetic-related genes varied among the control samples depending on the passage number. At P7, basal level expression was maintained during treatment, while it increased at P28 and decreased at P50. These results indicated that the cellular epigenetic status in C9 cells changed along the passages. 


\section{Discussion}

SP6 is an indispensable factor during tooth development ${ }^{9,10}$. However, the precise role and regulation of SP6 remain unclear. Recently, we found that SP6 is a short-lived protein and specifically degraded by the ubiquitin-independent $20 S$ proteasome pathway ${ }^{14}$. Thus, it is suggested that SP6 required fine-tuning in vivo to function in a time- and space-specific manner.
$\mathbf{A}$

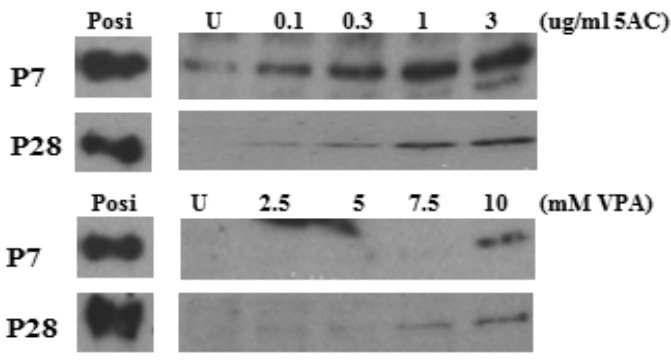

C

Exogenous Sp6
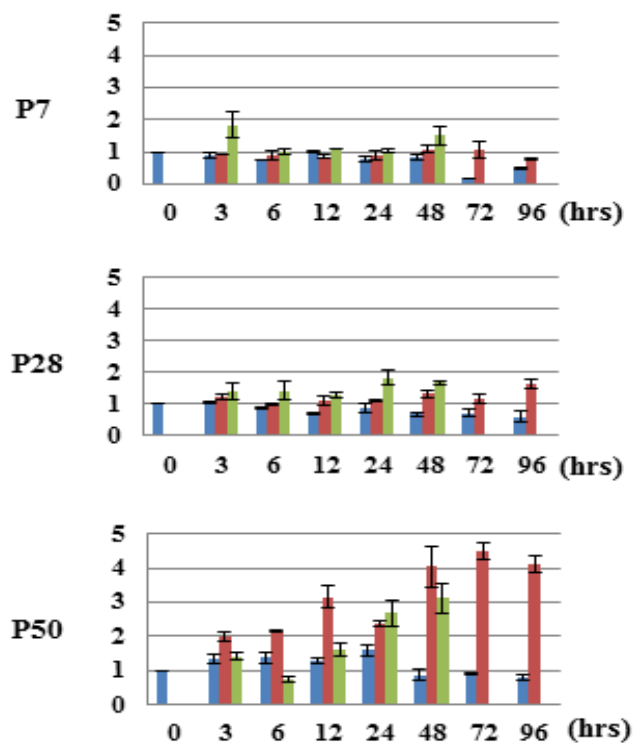

B
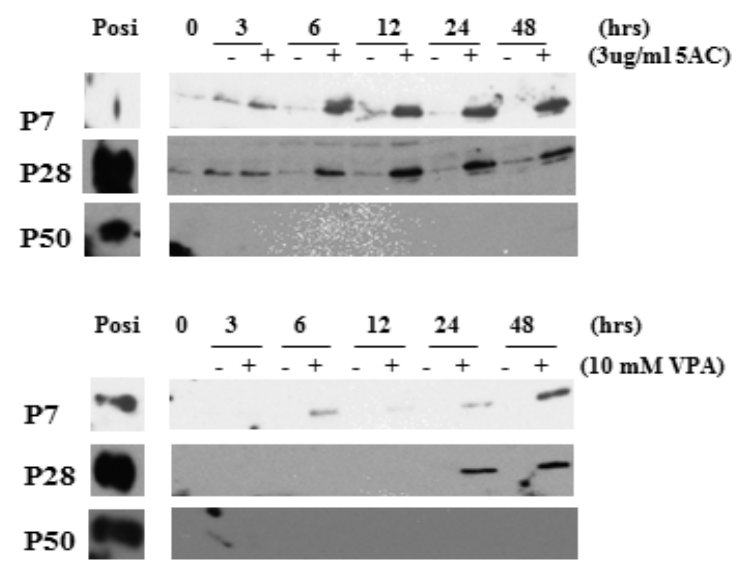

Endogenous Sp6
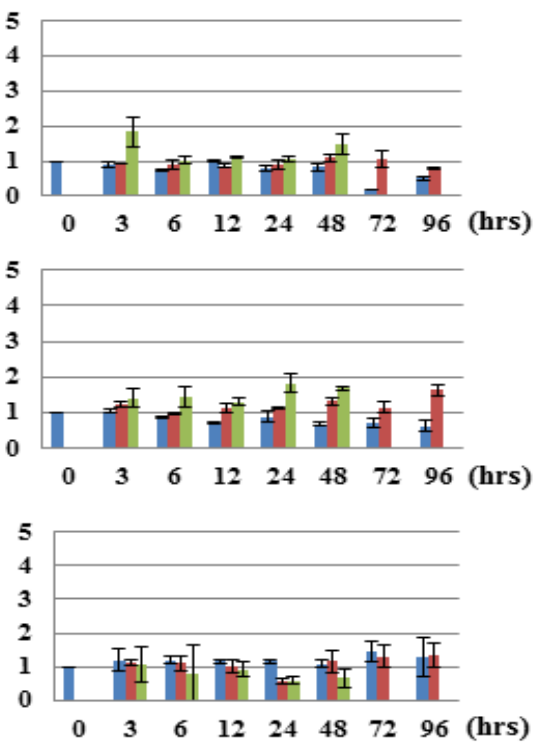

\section{Control $5 \mathrm{AC}$ VPA}

Figure 2. Effects of DNMTI and HDACl on Sp6 expression in C9 cells. (A) Dose-dependent effects of 5AC and VPA on SP6 expression in C9 cells at P7 and P28. Upper panel: cells were treated with 5AC. Lower panel: cells were treated with VPA. Posi: positive control; U: no treatment. (B) Time course analysis of 5AC and VPA treatment on SP6 expression in C9 cells. Upper panel: cells were treated with 5AC. Lower panel: cells were treated with VPA. Posi: positive control. (C) Time course analysis of 5AC and VPA treatment on exogenous and endogenous Sp6 mRNAs in C9 cells. Blue: control; red: 5AC treatment; green: VPA treatment. 
Previous reports revealed that DNA methylation and histone deacetylation play potential roles in the regulation of gene expression ${ }^{21,22}$. Gene silencing is not only strict for endogenous genes but also for exogenous DNA that is introduced into mammalian cells $^{18,23}$. The interplay between DNA methylation and histone modification is profoundly involved and they act synergistically during gene silencing, although it is unclear when and which epigenetic regulatory mechanism initiates first ${ }^{21,22}$. In addition, it has been reported that the CMV promoter, which is frequently used as one of the well-known strong mammalian promoters, can be silenced both by DNA methylation and histone modification such as deacetylation and methylation over time in culture $18,24,25$
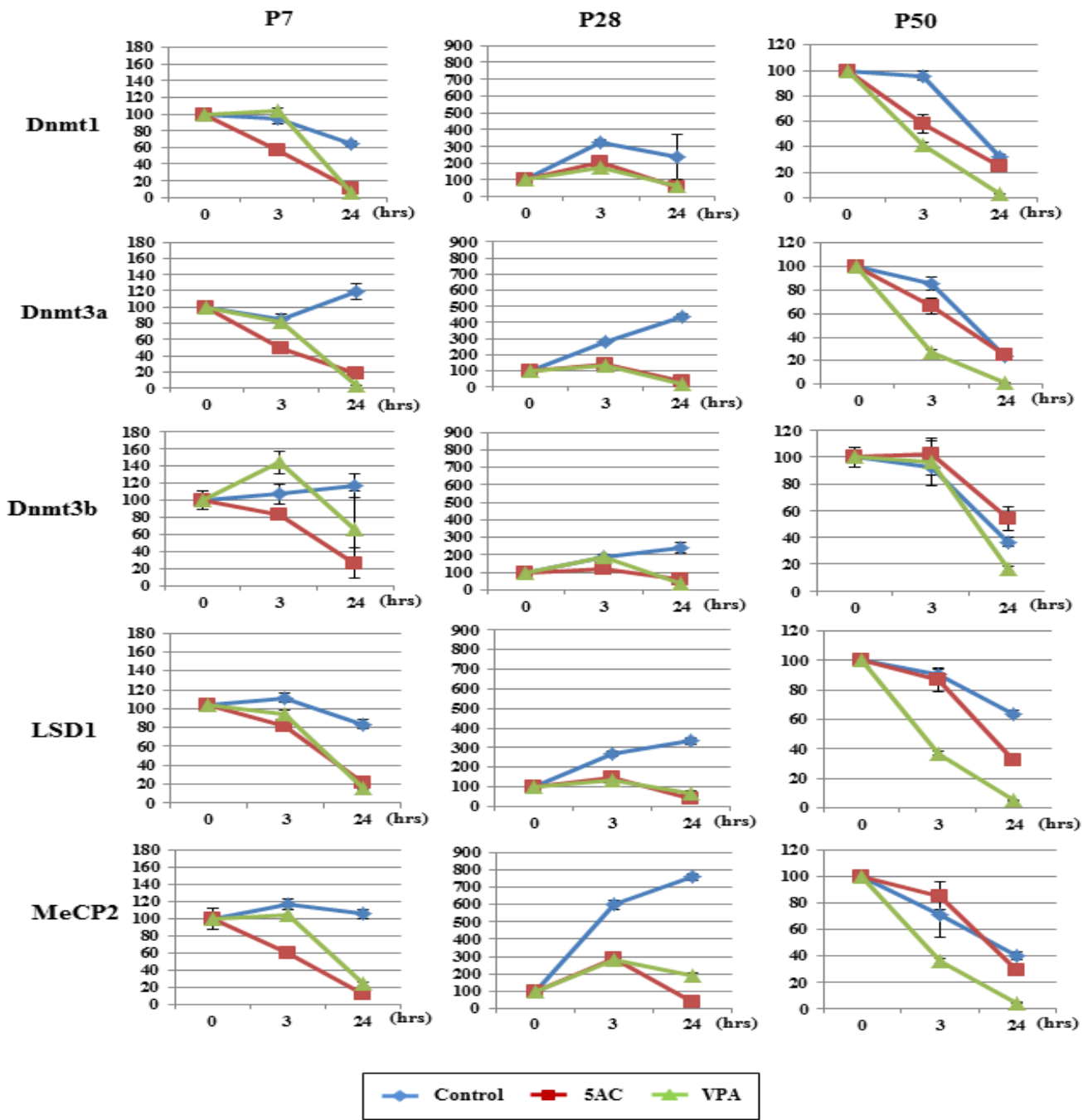

Figure 3. Analysis of epigenetic-related gene expressions. C9 cells at P7, P28, and P50 were treated with either 5AC or VPA for the indicated times. mRNA expressions of epigenetic-related genes (Dnmt1, Dnmt3a, Dnmt3b, MeCP2, and LSD1) were analyzed by qRT-PCR. Blue: control; red: 5AC treatment; green: VPA treatment. 
In our study, we demonstrated that SP6 protein expression in $\mathrm{C} 9$ cells was induced by both DNMTI and $\mathrm{HDACl}$ treatments at P7 and P28, while only exogenous Sp6 mRNA expression was enhanced at P50 by both treatments. These findings clearly indicated that SP6 protein and its MRNA expression were independently regulated in $\mathrm{C} 9$ cells by mechanisms that were different from the simple $\mathrm{CMV}$ promoter inactivation by DNA methylation. This suggests two important aspects of this regulation.

First, SP6 protein expression disappeared when the level of total Sp6 mRNA started to decrease, primarily because of reduced exogenous Sp6 mRNA (Figure 1A and 1B). This indicated that the translational efficiency was overwhelmed by degradation because of the nature of SP 6 with a short half-life or its high-sensitivity to $20 \mathrm{~S}$ proteasome activity ${ }^{14}$. Furthermore, SP6 protein was induced but not transcriptionally enhanced at P7 and P28 by treatments with $5 \mathrm{AC}$ and VPA (Figure $2 \mathrm{~A}$ and $2 B)$. This suggested effects of DNMTI and $\mathrm{HDACl}$ on either the translational efficiency of $S p 6$ mRNA, or the sensitivity of the SP6 protein to $20 \mathrm{~S}$ proteasome by SP 6 binding proteins as has been shown in the case of hypoxia-inducible factor- $1 \alpha$ stability, which is regulated by recruited $\mathrm{HDACl}$ through its binding with metastasis-associated protein $1^{26}$. Although not a simple problem, it is a quite interesting issue to examine whether SP6 protein stability is controlled by protein-protein interactions.

Second, we could not detect any responsiveness of endogenous Sp 6 mRNA expression to 5AC and VPA throughout the cell passages or that of exogenous Sp6 mRNA at P7 and P28 (Figure 2C). This finding indicated that the chromatin of the endogenous Sp6 gene locus was opened and that endogenous Sp6 gene was transcribed at a weak level; however, this level was not sufficient to produce a detectable level of SP6 protein. Thus, we need to consider several regulatory mechanisms, including the balance between transcriptional activators and repressors, mRNA stability, and translation initiation. In addition, a non-coding RNA, such as microRNA, may be involved, in which the non-coding RNA may block translation by cleaving the abundant exogenous Sp6 mRNA or inhibit the translational processes ${ }^{27}$. Because the reciprocal expression of Sp6 mRNA

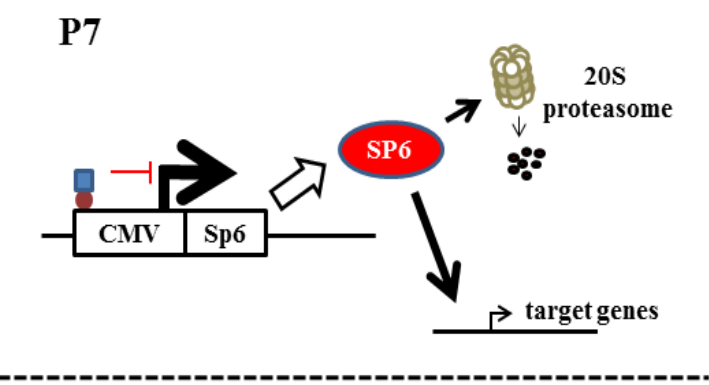

P28

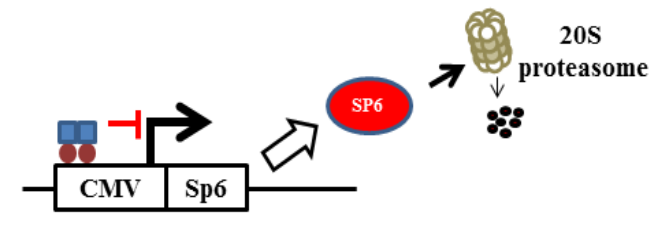

P50

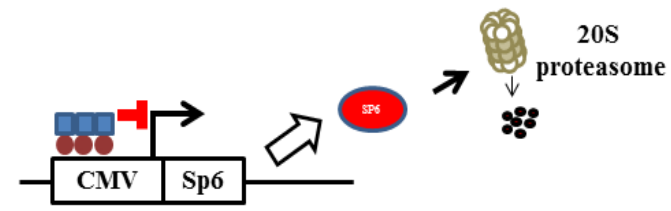

Figure 4. Working model of the $\mathrm{C} 9$ cell status leading to silencing of exogenous Sp6 gene during long-term culture. Detailed description of this model is in the Discussion. CMV: cytomegalovirus promoter; red circle: methylated CpG; blue rectangle: deacetylated histone.

and Sp6 opposite strand transcript (Sp6os) has been reported to be tissue-specific ${ }^{28}$, it will be interesting to examine whether Sp6os has a role in the expression of SP6 protein in C9 cells.

Furthermore, we also found that the responsiveness of epigenetic-related genes to $5 A C$ and VPA changed during long-term culture. In C9 cells at P7 and P28, both reagents significantly repressed epigenetic-related gene expressions compared to controls, while at P50 the levels of these genes exhibited no gross changes compared to controls, except for LSD1. Because DNA methylation is not required for maintenance or establishment of the silent chromatin state, the chromatin status at P50 was already supposed to be silenced by other mechanisms ${ }^{29}$. Moreover, we observed good inhibitory responses of the mRNA expressions of five epigenetic regulators to both $5 A C$ and VPA at P28, 
suggesting that C9 cells have epigenetic plasticity during long-term culture.

Taken together, we conclude that epigenetic regulation is involved in $5 p 6$ transgene expression in C9 cells. Based on our findings, we have hypothesized a working model that indicates the stepwise changes in cellular competency to activate Sp6 transgene expression in C9 cells; this model is outlined in Figure 4. During early passage (P7), the CMV promoter is weakly methylated but does not affect the transcriptional processes to a great extent. Therefore, strongly enhanced Sp6 mRNA is translated into SP6 protein and acts to regulate the target genes. Some parts of the SP6 protein pool are degraded by a proteasome pathway as shown previously ${ }^{14}$. During a middle passage (P28), moderate methylation of the CMV promoter region is implicated in the repression of $S p 6$ transcription, resulting in SP6 protein degradation. At a later passage (P50), hypermethylation of the CMV promoter region occurs upon long-term culture, which results in a limited copy number of Sp6 transcripts being translated and most of the SP6 protein products are degraded by a proteasome pathway.

\section{Conclusion}

We investigated the molecular mechanisms underlying Sp6 regulation through transgene silencing detected in Sp6 stable transformant C9 cells. Our findings provide new insights to analyze the regulation of SP6 during the developmental stages and its tissue-specificity in vivo.

\section{Acknowledgements}

This work was supported, in part, by Grants-in-Aid for Scientific Research (No.21791805), Cooperative Research Grant of the Institute for Enzyme Research, the University of Tokushima, and a Research Grant from KAO Health Science Research.

\section{References}

1. Thesleff I. 2003. Epithelial-mesenchymal signalling regulating tooth morphogenesis. J Cell Sci, 116:1647-1648.

2. Brook AH. 2009. Multilevel complex interaction between genetic, epigenetic and environmental factors in the aetiology of anomalies of dental development. Arch Oral Biol, 54 Suppl 1:S3S17.

3. Thesleff I. 2006. The genetic basis of tooth development and dental defects. Am J Med Genet A, 140:2530-2535.

4. Jimenez-Rojo L, Ibarretxe $G$, Aurrekoetxea $M$, de Vega S, Nakamura T, Yamada Y, and Unda F. 2010. Epiprofin/Sp6: a new player in the regulation of tooth development. Histol Histopathol, 25:1621-1630.

5. Suske G, Bruford E, and Philipsen S. 2005. Mammalian SP/KLF transcription factors: Bring in the family. Genomics, 85:551-556.

6. Scohy S, Gabant P, Van Reeth T, Herveldt V, Dreze PL, Van Vooren P, Riviere M, and Szpirer C. 2000. Identification of KLF13 and KLF14 (Sp6), novel members of the Sp6/XKLF transcription factor family. Genomics, 70:93-101.

7. Nakamura $T$, Unda F, de-Vega S, Vilaxa A, Fukumoto S, Yamada KM, and Yamada Y. 2004. The Kruppel-like factor epiprofin is expressed by epithelium of developing teeth, hair follicles, and limb buds and promotes cell proliferation. J Biol Chem, 279:626-634.

8. Bei M. 2009. Molecular genetics of ameloblast cell lineage. J Exp Zool B Mol Dev Evol, 312B:437444.

9. Nakamura $T$, de Vega $S$, Fukumoto S, Jimenez L, Unda F, and Yamada T. 2007. Transcription factor epiprofin is essential for tooth morphogenesis by regulating epithelial cell fate and tooth number. J Biol Chem, 283:4825-4833.

10. Hertveldt $V$, Lourya $S$, Van Reeth $T$, Drèze $P$, Van Vooren P, Szpirer J, and Szpirer C. 2008. The development of several organs and appendages is impaired in mice lacking Sp6. Dev Dyn, 237:883892.

11. Ruspita I, Miyoshi K, Muto T, Abe K, Horiguchi T, and Noma T. 2008. Sp6 down regulation of follistatin gene expression in ameloblast. $J$ Med Invest, 55:87-98. 
12. Wahyudi IA, Horiguchi T, Miyoshi K, Muto T, Utami TW, Hagita H, and Noma T. 2010. Isolation and characterization of mouse specificity protein 6 promoter. Indones J Den Res, 1:21-34.

13. Miyoshi K, Nagata H, Horiguchi T, Abe K, Wahyudi IA, Baba Y, Harada H, and Noma T. 2008. BMP2-induced gene profiling in dental epithelial cell line. J Med Invest, 55:216-226.

14. Utami TW, Miyoshi K, Hagita H, Yanuaryska RD, Horiguchi T, and Noma T. 2011. Possible linkage of SP6 transcriptional activity with amelogenesis by protein stabilization. J BiomedBiotechnol, doi:10.1155/2011/320987.

15. Zhu WG, Dai Z, Ding H, Srinivasan K, Hall J, Duan W, Villalona-Calero MA, Plass $\mathrm{C}$, and Otterson GA. 2001. Increased expression of unmethylated CDKN2D by 5-aza-2'-deoxycytidine in human lung cancer cells. Oncogene, 20:7787-7796.

16. Marks PA and Xu WS. 2009. Histone deacetylase inhibitors: Potential in cancer therapy. J Cell Biochem, 107:600-608.

17. Abe K, Miyoshi K, Muto T, Ruspita I, Horiguchi T, Nagata T, and Noma T. 2007. Establishment and characterization of rat dental epithelial derived ameloblast-lineage clones. J Biosci Bioeng. 103:479-485.

18. Grassi G, Maccaroni P, Meyer R, Kaiser H, Ambrosio ED, Pascale E, Grassi M, Kuhn A, Nardo PDi, Kandolf R, and Kupper JH. 2003. Inhibitors of DNA methylation and histone deacetylation activate cytomegalovirus promoter-controlled reporter gene expression in human glioblastoma cell line U87. Carcinogenesis, 24:1625-1635.

19. Nan X, Campoy FJ, and Bird A. 1997. MeCP2 is a transcriptional repressor with abundant binding sites in genomic chromatin. Cell, 88:471-481.

20. Forneris F, Binda C, Vanoni MA, Battaglioli E, and Mattevi A. 2005. Human histone demethy- lase LSD1 reads the histone code. J Biol Chem. 280:41360-41365.

21. Ben-Porath I and Cedar H. 2001. Epigenetic crosstalk. Mol Cell, 8:933-935.

22. Vaissière T, Sawan C, and Herceg Z. 2008. Epigenetic interplay between histone modifications and DNA methylation in gene silencing. Mutat Res, 659:40-48.

23. Chen WY, Bailey EC, McCune SL, Dong JY, and Townes TM. 1997. Reactivation of silenced, virally transduced genes by inhibitors of histone deacetylase. Proc Natl Acad Sci USA, 94:57985803.

24. Hsu CC, Li HP, Hung YH, Leu YW, Wu WH, Wang FS, Lee KD, Chang PJ, Wu CS, Lu YJ, Huang THM, Chang YS, and Hsiao SH. 2010. Targeted methylation of CMV and E1A viral promoters. Biochem Biophys Res Commun, 402:228-234.

25. Mehta AK, Majumdar SS, Alam P, Gulati N, and Brahmacari V. 2009. Epigenetic regulation of cytomegalovirus major immediate-early promoter activity in transgenic mice. Gene, 428:20-24.

26. Yoo YG, Kong G, and Lee MO. 2006. Metastasisassociated protein 1 enhances stability of hypoxia-inducible factor-1 alpha protein by recruiting histone deacetylase 1.EMBO J, 25:1231-1241.

27. Egger G, Liang G, Aparicio A, and Jones PA. 2004. Epigenetics in human disease and prospects for epigenetic therapy. Nature, 429:457-463.

28. Herveldt V, De Mees C, Scohy S, Van Vooren P, Szpirer J, and Szpirer C. 2007. The Sp6 locus uses several promoters and generates sense and antisense transcripts. Biochimie, 89:1381-1387.

29. Feng YQ, Desprat R, Fu H, Olivier E, and Lin CM. 2006. DNA methylation supports intrinsic epigenetic memory in mammalian cells. Plos Genetics, 2:e65 doi:10.1371/journal.pgen.0020065. 